\title{
Job shop configuration optimization at Tinker Air Force Base
}

\author{
L. Leemis, A. Badiru, B.L. Foote, A. Ravindran \\ School of Industrial Engineering \\ The University of Oklahoma \\ 202 West Boyd, Room 124 \\ Norman, OK 73019
}

\author{
Larry Williams \\ Tinker Air Force Base \\ Oklahoma City, OK 73170
}

This paper discusses a large simulation model that was developed to aid the reconstruction efforts after a disastrous fire at Tinker Air Force Base. The model, developed in SLAM, facilitated the analysis and efficient design of the functional layout that replaced the previous machine-based layout in the appropriate number of machines to place within a modular repair center (MRC), stacker capacity for in-process inventory, location of elevators for sending parts to the conveyor, and the optimal design and routing scheme for overhead conveyor system. The new layout, as predicted by the simulation model, has proven to be quite effective compared with the prefire layout. Improvements that have been achieved include a 50 to $80 \%$ decrease in material handling, substantive decrease in flow times, better management control of part transfers, $\$ 4.3$ million savings from the elimination of excess capacity, and $\$ 1.8$ million savings resulting from higher direct labor efficiency.

\section{Introduction}

Tinker Air Force Base is the primary engine overhaul facility for the U.S. Air Force. A fire in the Fall of 1984 prompted a reevaluation of the layout of the overhaul facility. Tinker manufacturing analysts, assisted by staff from the University of Oklahoma, used simulation to design the Modular Repair Centers and to evaluate the impact of a transition from the previous machine based layout to the proposed functionally based layout.

Prior to the fire, the facility was organized by placing similar operations (e.g., grinding, welding, painting) together, which was effective for minimizing queueing time for parts, but inefficient in terms of material handling, flow time organizational transfers. For example, the material handling for a diffuser case was 9 linear miles prior to the fire, flow time for a combustion can was 32 days, and the number of organizational changes for a part ran as high as 30 to 50 times. These organizational changes for a part created management problems, since it was difficult to determine responsibility for work on a particular part.

Since pre-fire experimentation with a consolidation of the combustion can workload into a self-contained shop proved successful, it was decided to reconfigure the whole facility according to the Modular Repair Center (MRC) concept upon recovering from the fire damage. An MRC, or "shop within a shop", accepts parts with similar geometrics and industrial processes in order to reduce flow time and material handling, as well as provide single point organizational responsibility and control.

The maintenance facility overhauls approximately 1500 different jet engine parts identified by a Work Control Document (WCD). The annual WCD volume is approximately one million parts. It was proposed to divide the 
facility into $13 \mathrm{MRC}$ 's (e.g., nozzels, seals, gear box) according to the geometry and size of parts. Three operations, plating, painting and heat treat, could not be transferred to each MRC due to cost or safety limitations; so some routing out of an MRC was inevitable for certain parts. This external routing of parts was accounted for in the simulation by adding appropriate time delay factors to the processing of the parts affected.

\section{The Model}

The simulation model, called Tinker Integrated Planning and Simulation (TIPS), is written using the discrete event orientation in SLAM (Pritsker, 1986) and contains approximately 1750 lines of FORTRAN code. A survey of existing software for manufacturing applications is given in Law and Haider (1989). The entities in the model are the WCD's flowing through one particular MRC. Features of the TIPS include three shifts, machine down time, worker absenteeism, transfer to other MRC operations (i.e., painting, plating and heat treat) and stackers to model WCD storage when machine queue lengths are exceeded. The simulation model is capable of storing 70,000 entities (concurrent WCD's) in an MRC. Despite this, three of the MRC's were so large that they had to be broken into smaller family groups.

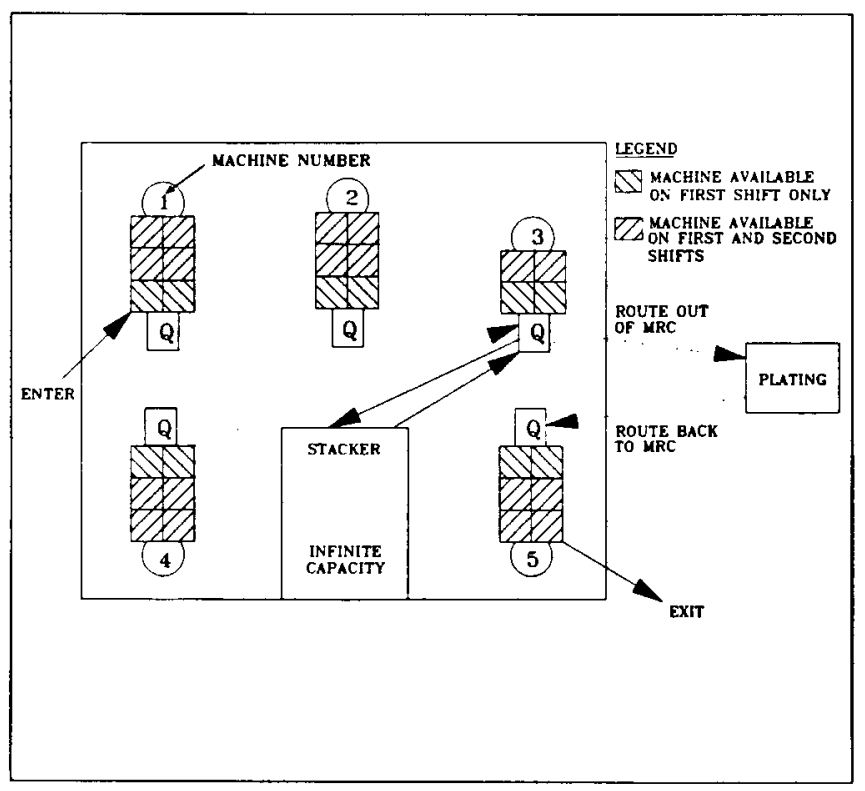

Figure 1. Sample MRC Configuration

Figure 1 shows a simplified diagram of a five machine MRC. A WCD that enters at the left side of the diagram has a 1-4-3-plating-5 machine sequence. A stacker is included in the model since machines only have limited queue sizes, and the infinite capacity stacker is used for temporary storage when there is an overflow. The figure shows such a transfer for machine number three, which has only four machines and a small queue capacity. The route out to plating and return includes an 8-hour material handling delay. The cross hatching on the machines in the diagram indicates the shifts where they are available. For example, there are six machines of type number one available during the day shift, and only four are available during the second shift. If a WCD is on a machine when the shift change occurs, it is assumed that the machine completes processing the WCD prior to becoming idle due to decreased labor capacity on the next shift.

The data used to determine the rate of flow of WCD's through each MRC was obtained from Tinker Air Force Base. The data for each MRC came in two sets, the 1985 Fiscal year data and the 2000 engine equivalents (when the facility runs at full capacity) data. Both data sets contained a list of the WCD's for the MRC, the operation sequence for each WCD, the corresponding machine process time for each WCD, the corresponding standard labor time for each WCD, the UPA (units per assembly) number for each WCD and a vector containing the relative frequencies of each WCD. In addition, the projected size (e.g. number of machines of each type) of each MRC and data for the calculation of a FROM-TO matrix (for inter- and intra-MRC transfers) was contained in the data. All data was transformed to a format that allowed SLAM to execute the discrete event model.

Two features of the TIPS simulation model make it particularly unique. First, the model was so large that it used the SLAM language at its maximum configuration to run a single MRC. The research team had to consult with Pritsker \& Associates to find out how Slam's limits could be extended in the source code. Second, the model integrated both physical (machines) and skill (labor) resources in a single model that supported a bottleneck analysis, space analysis and overhead conveyor routing analysis. The model was designed for managers, and two hands-ontraining sessions were held at Tinker AFB to allow managers to use TIPS for decision making.

Some features of the model required special considerations as the OU team constructed the model of the proposed shop configuration:

* Down time.

Machine breakdown is a factor that affects the flow time and throughput for an MRC. After each machine completes processing on a part, a breakdown occurs with probability that depends on the machine. The time to repair a machine is assumed to be exponentially distributed since only the mean repair time is available as input data. The distributions and parameters used in the simulation were based on estimates by Tinker AFB personnel.

* Interarrival and service time distribution. Since no data was available on the interarrival distribution, deterministic interarrival and service times were used based on the annual volume of that particular WCD.

* Labor utilization.

The modeling of a WCD being serviced on a machine is difficult because both a machine and an operator are required to service a part. Each WCD requires an operator and a machine for durations determined from data before completing processing on that machine. In addition, sick leave, training leave and vacations for machine operators are modeled. 


\section{Calculation of Number of Machines for $95 \%$ Availability}

The simulation model provides a utilization histogram giving the percent of time that a particular machine is busy. Because only one shift is worked in the FY 85 models, machines are idle during the second and third shifts. As a result, the statistics for idle machines ( $0 \%$ utilization) are distorted. To overcome the distortion, some calculation adjustment was performed to achieve $95 \%$ machine availability. The adjustment procedure consisted of the four steps shown below:

Step 1: Multiply the individual relative frequencies in the utilization histogram by 3 to account for the idle second and third shifts.

Step 2: Sum the tripled frequencies cumulatively from the last row (frequency data in the last cell).

Step 3: Stop when the cumulative sum is greater than 0.05 .

Step 4: Read across to the corresponding cell upper limit. This upper limit is the number of machines to place in the MRC to achieve $95 \%$ utilization.

An example of the above procedure is shown below for one of the machines in MRC CC2 (Combustion Can).

Table 1. Frequency Distribution of Machine Utilization

$\begin{array}{cccc} & \text { Rel. Freq. } & \text { Cum. Freq. } & \text { Upper Cell Limit } \\ & 0.683 & 0.683 & 0 \\ & 0.115 & 0.798 & 1 \\ & 0.068 & 0.866 & 2 \\ & 0.048 & 0.915 & 3 \\ & 0.035 & 0.950 & 4 \\ & 0.022 & 0.972 & 5 \\ & 0.014 & 0.986 & 6^{*} \\ & 0.009 & 0.995 & 7 \\ & 0.001 & 0.996 & 8 \\ & 0.003 & 0.999 & 9 \\ & 0.001 & 1.000 & 10 \\ & 0.0 & 1.000 & 11\end{array}$

Step 1/Step 2:

$3(0.0)+3(0.001)+3(0.003)+3(0.001)+3(0.009)+3(0.014)=0.084$

Step 3: $0.084>0.05$

Step 4: Required number of machines $=6$

\section{Problems With Large MRC's}

Some MRC's were too large to be handled by the simulation model. This led to excessively long simulation run times. As a result, the large MRC's had to be broken into smaller family groups. For example, the Combustion Can Shop (MRC C) was broken down into six families. TIPS can handle approximately 70,000 entities (or WCD's) at one time. The run times varied widely depending on the size of the MRC. For example, MRC GX 2000 engine equivalents data (i.e. estimated repair workload for the year 2000) took approximately one hour to run on an IBM 3081. The run times on a VAX 11/780 were generally eight times longer than the IBM run times. In one specific case, MRC CC2, one of the smallest families in MRC C, took 1.5 minutes to run on the IBM and 7.8 minutes on the VAX. The CC2 shop contains a maximum of 5 WCD's and can handle up to 63 different processes. It has an annual workload of about 821 parts. By comparison, the GX MRC handles about 329 WCD types and has up to 72 different organic processes. Its annual repair volume is over 100,000 parts. A warm-up period of 13 weeks (1 quarter) was used for each simulation run. Statistics on MRC's were collected starting with the fourteenth week. Simulation outputs were printed in 13-week time intervals to match regular production runs at Tinker AFB.

\section{Output}

The output from TIPS consisted of two parts: the standard SLAM summary report and a custom printout generated by a FORTRAN subroutine. The custom output helped in presenting the SLAM output in a format and level of detail suitable for prompt managerial decision making. The statistics presented in the output included the following:

1. Machine availability by shift for each process.

2. Maximum queue length in front of each process.

3. Average processing time.

4. Average waiting time for each process.

5. Number of units for each WCD type arriving to and leaving the system.

6. Part flow (in units) to and from the stacker with respect to each process.

7. Utilization level of each process per shift.

8. Total time in the system for each WCD. This included waiting time, handling time, processing time, and labor time.

9. Time spent in the stacker waiting for a specific process.

Supplementary FORTRAN programs were written to generate certain input data for the TIPS program. For example, a bottleneck program was used to set a minimum number of machines available for each process. Likewise, some supplementary programs used some of the SLAM output to generate other outputs that were of specific interest to management. For example, the COPT (Conveyor Optimization) program uses some of the SLAM output to generate an improved design for the overhead conveyor system using a shortest route model.

The TIPS program is, in effect, the nucleus of an integrated system of management decision aids. First, TIPS was originally used to determine the appropriate number of machines to place in each of the MRCs. Second, the use of the two data sets (FY 85 and 2000 engine equivalents) allows management to determine the process capability for the overhaul facility. Third, since individual parts are modeled as entities, the process plan for the overhaul of a particular 
part can be studied. Altering this process plan might possibly reduce bottlenecks or reduce flow time. Finally, the model can be used on-line to determine whether certain processes should be run on more than one shift, more machines of a particular type should be purchased, etc.

\section{Verification and Validation}

The simulation model was verified to determine if it was working as intended (Law and Kelton, 1982). Two verification techniques were used. First, the model was developed incrementally. This made it easier to debug the programs, and check to assure that each module was working correctly. Second, the outputs of each modular component of the overall model were analyzed for:

1. Reasonableness check (does the simulation seem to represent what actually goes on at Tinker AFB?)

2. Consistency check (does the output remain about the same for similar inputs?)

3. Run time check (does the program run longer than expected for a particular MRC?)

4. Output check (a 10\% increase in load should show more than a $10 \%$ increase in waiting time.)

Validation concerns a diagnostic check of how closely the simulation model matches the actual system. This was done by cross checking and model assumptions. For example, is the assumption of normally distributed processing times correct? Also, the simulation output was compared to historical data using a representative MRC simulation, with respect to average and range of the statistics collected.

\section{Conclusions}

The decision to transform the engine overhaul facility from the pre-fire layout to the MRC layout has proven to be an effective one. There has been a 50 to $80 \%$ decrease in material handling for long flow items, including a decrease from 9 linear miles to 3 linear miles for the diffuser MRC. This change in material handling decreased the flow time in the Combustion Can MRC from 32 to 19 days. Management control at a single point for a part is now possible due to a 50 to $70 \%$ decrease in organizational transfers for long flow parts. A savings of 30,000 square feet, $\$ 4.3$ million in excess machinery, and $\$ 1.8$ million in direct labor efficiency have resulted from the new layout.

The analysis to aid the transition to the MRC layout included the TIPS simulation program, overhead conveyor system design (Ravindran, et al., 1988). The TIPS program proved valuable in aiding the transition to the new layout by estimating performance measures (e.g. flowtime, queue statistics) that helped determine the number of machines of each type to place in an MRC. In one particular instance, the Nozzle MRC, the Production \& Engineering Department at Tinker indicated that there should be 24 workstations of a particular type. The TIPS analysis indicated that between 11 and 13 workstations should be placed in the Nozzle MRC, and this has proved to be sufficient. The transition to the $\mathrm{MRC}$ layout was effective for the engine overhaul facility at
Tinker AFB due to similarities in the geometries of certain parts, part mix, and the size of the facility. Simulation proved to be a valuable tool in assessing the effectiveness of the transition and determine appropriate parameters for each new MRC.

\section{Acknowledgement}

The authors would like to express their appreciation to Margarita Beneke, K. Janakiraman, Wan-Seon, Doug Stewart and Murali Subramaniam for their assistance in developing and running the TIPS model.

\section{References}

Foote, B.L., Ravindran, A., Badiru, A., Leemis, L. and Williams, L. (1988), "Simulation and network analysis in conveyor system design", Industrial Engineering, 20, 6, 48-53.

Law, A. and Kelton, D. (1982), Simulation Modeling and Analysis, McGraw-Hill.

Law, A. and Haider, S. (1989), "Selecting Simulation Software for Manufacturing Applications, Practical Guidelines \& Software Survey", Industrial Engineering, 31, 5, 33-46.

Pritsker, A.A.B. (1986), Introduction to Simulation and SLAM II, Third Edition.

Ravindran, A., Foote, B.L., Badiru, A., Leemis, L. and Williams, L. (1988), "Mechanized Material Handling Systems Design and Routing", Computers and Industrial Engineering, 14, 3, 251-270.

Ravindran, A., Foote, B.L., Badiru, A., Leemis, L. and Williams, L. (1989), "An Application of Simulation and Network Analysis to Capacity Planning and Material Handling Systems at Tinker Air Force Base," Interfaces, 19, 1, 102-115.

Lawrence Leemis is an associate professor in the School of Industrial Engineering at the University of Oklahoma. He received his BS and MS degrees in Mathematics, and his $\mathrm{PhD}$ in Operations Research from Purdue University. His research interests are in reliability and simulation.

Bobbie L. Foote is a professor in the School of Industrial Engineering at the University of Oklahoma. He received his BS, MA in Mathematics, and his $\mathrm{PhD}$ in Industrial Engineering from the University of Oklahoma. His research interests are in applied operations research, plant and production planning, inventory models/MRP, and quality control/assurance.

A. Ravindran is a professor in the School of Industrial Engineering at the University of Oklahoma. He received his BS in Electrical Engineering, and his $\mathrm{MS}$ and $\mathrm{PhD}$ in Industrial Engineering from the University of California at Berkeley. His research interests are in operations research, multicriteria optimization, energy systems, health planning models, and expert systems.

Adedeji Badiru is an assistant professor in the School of Industrial Engineering at the University of Oklahoma. He received his BS and MS in Industrial Engineering and also an MS in Mathematics from Tennessee Technological University and his PhD in Industrial Engineering from the University of Central Florida. His research interests are in manufacturing systems, project management, operations research, expert systems and simulation.

Larry M. Williams is the Chief of the Facilities and Equipment Branch of the Oklahoma City Air Logistics Center. His BS degree is in Mathematics and Physics from East Central State University and his MS degree is in Industrial Engineering from the University of Oklahoma. 